\title{
An Efficient Coding Method for Teleconferencing Video and Confocal Microscopic Image Sequences
}

\author{
Vinay Arya ${ }^{1}$, Ankush Mittal ${ }^{1}$, Amit Pande ${ }^{2}$ and R. C. Joshi ${ }^{1}$ \\ ${ }^{1}$ Dept. of Electronics and Computer Engineering, IIT Roorkee, Uttaranchal, India \\ ${ }^{2}$ Dept. of Electrical and Computer Engineering, lowa State University, Ames, USA
}

In this paper we propose a three-dimensional vector quantization-based video coding scheme. The algorithm uses a $3 \mathrm{D}$ vector quantization pyramidal codebook-based model with adaptive pyramidal codebook for compression. The pyramidal codebook-based model helps in getting high compression in case of modest motion. The adaptive vector quantization algorithm is used to train the codebook for optimal performance with time. Some of the distinguished features of our algorithm are its excellent performance due to its adaptive behavior to the video composition and excellent compression due to codebook approach. We also propose an efficient codebook-based post-processing technique which enables the vector quantizer to possess higher correlation preservation property. Based on the special pattern of the codebook imposed by post-processing technique, a window-based fast search (WBFS) algorithm is proposed. The WBFS algorithm not only accelerates the vector quantization processing, but also results in better rate-distortion performance.

The proposed approach can be used for both teleconferencing videos and to compress images obtained from confocal laser scanning microscopy (CLSM). The results show that the proposed method gave higher subjective and objective image quality of reconstructed images at a better compression ratio and presented more acceptable results when applying image processing filters such as edge detection on reconstructed images. The experimental results demonstrate that the proposed method outperforms the teleconferencing compression standards H.261 and LBG-based vector quantization techniques.

Keywords: confocal microscopic images, codebook, codebook-preprocessing, rate distortion, vector quantization, window-based fast search

\section{Introduction}

Vector quantization has been widely used in image and video compression because of its superior rate distortion performance over traditional scalar quantization schemes and its simple decoder structure. This method of compression has gained interest over conventional means of compression (i.e., DPCM and Transform coding [1]) because the conventional methods usually employ some form of scalar quantization. Scalar quantization is not optimal as the successive samples in a digital signal are usually correlated or dependent. A vector can be used to describe almost any type of pattern, such as the patterns that arise in digital images.

Different approaches may be considered for video compression. Since lossless compression approaches result in small compression ratios, we have considered the lossy compression techniques. Several studies dealing with vector quantization-based lossy compression of images has been proposed and applied to different image and videos [2-8].

Some of the main approaches developed are discussed in this section. Localized model-based approach to teleconferencing using three-dimensional vector quantization [2] method uses LBG algorithm for creating a codebook. This codebook creating method does not vary with time. Thus this codebook needs to be trained for optimal performance with varying time, and hence varying input vector characteristics. To overcome this shortcoming, we have proposed an adaptive codebook approach.

Besides good R-D tradeoff, a good encoder should also posses an acceptable level of complexity. Predictive Vector Quantizer [3] and Finite State Vector Quantizer [4] alleviate the computational burden of a vector quantizer with 
memory, but at the same time they utilize the correlation among neighboring vectors. In [5], the inter-index correlation is exploited to code the indices more efficiently. Nevertheless, the easiest way to exploit the inter-vector correlation is to use the DPCM technique in the index domain, i.e., to precede the entropy coding of indices with a DPCM stage. Motivated by these considerations, a simple but efficient post-processing technique for adaptive codebook is proposed. The post-processing is done to reorder the codebook according to the potential of code words. The reordering is done once for all and is applicable to all existing codebook design methods. The post-processed codebook possesses a special structure which in turn leads to decreased first-order differential entropy, i.e., increased correlation between successive indices. This is beneficial to subsequent DPCM and entropy coding, and it improves the performance of the entire coding system. Another merit of the proposed post-processing techniques is that it imposes a certain structure to the codebook. This structure can be utilized to accelerate the searching process. Thus it relieves the computational requirement of the encoder.

For microscopic medical images, Burt [6] and Adelson [7] introduced quantization into the image pyramid structure and proposed a multiresolution lossy compression technique using a scalar quantizer. In 2003, Cockshott proposed a vector quantization-based $3 \mathrm{D}$ pyramid approach [8]. This method has 3D codebooks of size $2 \times 2 \times 4$. By using multiple level localized codebooks with variable size, it is possible to get high compression even if there is some motion in microscopic image sequence.

\section{Confocal Microscopic Image Sequence}

Confocal laser scanning microscopy (CLSM), which is single photon microscopy, has been available to biomedical scientists for almost 20 years [9]. However, it is only in very recent times that affordable computer power has enabled biologists to fully exploit the data contained within the large $(>100 \mathrm{Mb})$ image volumes. CLSM is used to collect 3D volumetric data describing the cellular organization and receptor protein distribution through the vascular wall of small segments of human and animal arteries.
Briefly, arterial segments are stained with fluorescent markers for the cell nucleus or betaadrenergic receptors. Tissues are then slide mounted on the stage of either a NORAN3 (nuclear work) or Leica4 (receptor work) CLSM. Serial optical sections $(x, y$ plane) are collected at intervals of $1 \mu \mathrm{m}$ down through the axial plane (z-axis) to produce a 'stack' of optical planes which can be processed as a 3D volume. The processing, analysis and transfer of the resulting data volumes are time inefficient. A robust non-lossy or low distortion lossy compression routine would be of great value for biomedical purpose, e.g. in studying vascular structure [10]. Furthermore, the emergence of multi photon microscopy as a practical laboratory tool now enables even greater depth penetration within thick biological samples. This, coupled with the studies involving multiple fluorophores imaged over time period, results in the collection of data sets approaching 1 GB per experiment. Without efficient compression algorithms, management of such enormous amounts of data will be very difficult.

Three-dimensional image compression methods outperform their two-dimensional counterparts in the sense of higher rate distortion performance for compressing volumetric image data. The state-of-the-art transform-based 3D compressors, such as 3D-SPIHT and 3D-DCT, are characterized by their rate control ability, where the qualities of the image are adjustable with respect to the rates, but are not explicitly controllable. In [8], the authors discuss the differences between 3D microscopy raw data and the movie sequence. In microscopy the data corresponds to tracer densities at sub volumes in a 3D grid, with the size of the sub volumes constrained by the microscopy optics. This contrasts with the data in a movie sequence which has time varying $3 \mathrm{D}$ generating surfaces. In $[8]$, the authors hypothesize that the higher order statistics of 3D microscopy data will differ from those of film and that the optimal compression strategy will differ from that used in film and video applications.

In [8], the author presents the use of 3D pyramid data structures to compress microscopy data. In [11], the author presents vector quantizationbased method in an enhanced image pyramid 
with error feedback so that the quality of the decompressed image depends only on the encoding of coefficients from the finest band. This achieves some effective reduction of artifacts.

In 3D microscopy, the raw data corresponds to tracer densities at sub volumes in a 3D grid with the size of the sub volumes constrained by the microscopy optics. The data is typically digitized as a sequence of $2 \mathrm{D}$ images i.e., a three dimensional array with time, $x$ and $y$ axis as the three dimensions [8].

The paper is organized as follows. The vector quantization and adaptive codebook procedures are explained in Section 2. 3D vector quantization used for compression and encoding of teleconferencing videos and confocal microscopic image sequences is described in Section 3. An efficient codebook post processing technique and a window-based fast search algorithm for vector quantization are explained in Section 4. The experimental results and analysis are given in Section 5. Section 6 gives conclusions and future work.

\section{Vector Quantization and Code Book}

Vector quantization (VQ) is used in sophisticated digital signal processing where in most cases the input signal already has some form of digital representation and the desired output is a compressed version of the original signal. VQ is usually employed for data compression purposes. A vector quantizer $Q$ of dimension $k$ and size $N$ is a mapping from a vector in $k$ dimensional Euclidean space, $R^{k}$, into a finite set $C$ containing output or reproduction points that exist in the same Euclidian space as the original point. These reproduction points are called code vectors or code words and this set forms a codebook $C$ with $N$ distinct code words. Thus the mapping function $Q$ is defined as:

$$
Q: R^{K} \rightarrow C
$$

A vector quantizer can be decomposed into two components, the vector encoder and the vector decoder and likewise index, $I$, and a quantized output value, $Q(x)$. The decoder is sometimes referred to as an "inverse quantizer". Associated with every $N$ point, vector quantizer is a partition of $R^{k}$ into $N$ regions or cells, and each cell has an index. The encoder gives an index to the given vector. On the other end, the decoder generates an output vector from a given index. The task of the encoder is to identify the location of input vector in one of the $N$ geometrically specified regions of $k$ spaces. Decoding is simply a table lookup task fully determined by specifying the codebook. A decoder does not need to know the geometry of the partition to perform his job. Vector quantization has been widely used in image-coding systems because of its superior rate distortion (R-D) performance over traditional scalar quantization schemes and simple decoder structure.

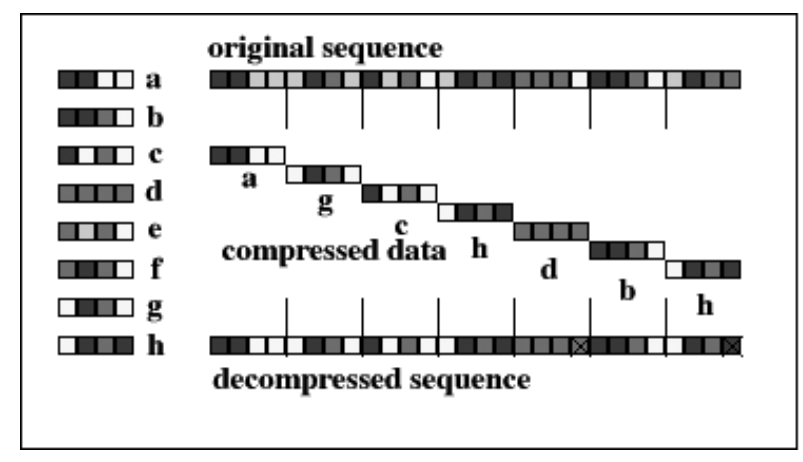

Figure 1. Vector quantizer.

The encoder and the decoder can be separately modeled with a corresponding primary structure. Let $A$ denote the index generator, as the mapping $A: B_{N} \rightarrow J$ and its inverse, the index decoder, $A^{-1}:\left(b_{1}, b 2, \ldots, b_{N}\right)$ with and $b$ is restricted to have exactly one nonzero component. Then $A(b)=I$ if $b_{i}=1$ and $b_{j}=0$ for $j \neq i$. Then the encoder operation can be written as:

$$
E(x)=A\left(S_{1}(x), S_{2}(x), S_{3}(x), \ldots S_{N}(x)\right)
$$

and the corresponding decoder operation is given by:

$$
D(i)=\sum_{i=1}^{N} y_{i} A^{-1}(i) .
$$

\section{The Codebook}

Different available methods of creating codebooks essentially do the same basic tasks. For $L$ codebook entries, the $M$-dimensional vector space is sectioned into $L$ non-overlapping cells. 
Let $C_{i}$ be the centroid of the training vectors within cell $i$. The centroid is the multidimensional mean of the training vectors for a particular cell. The centroid of the cells represents the output code vector associated with the corresponding cells. In other words, during the encoding process when an input vector falls within a particular cell, the index of that cell will be transmitted as the codeword. For the decoding process, the centroid of the cell will be output vector [12]. The best codebook for a given set of training data is the one which will minimize the quantization error over the vector to be quantized. However, even for moderately sized codebooks, an exhaustive search for the best codebook results in an impractically large number of computations. So, codebooks are generated or trained using statistical clustering algorithms. The LBG algorithm named after Linde, Buzo and Gray, is a widely used clustering method for generating a codebook. The algorithm begins with an initial set of codebook vectors, assigns the training vectors to the nearest codebook vector, then re-computes the codebook vector as the centroid of all the vectors assigned to that codebook entry. The process is repeated until convergence, or till there is no reduction in the overall quantization error [13].

\section{Adaptive VQ Codebook}

The codebook discussed above (LBG) does not vary with time. We have used a time varying approach because of its ability to adjust its codebook vectors as new pattern becomes available, so as to accurately model the class representation of the pattern. It is extremely important to train this codebook for optimal performance with varying time, and hence with varying input vector characteristics.

The proposed algorithm is adaptive with time. For each new vector we search for the nearest existing vector in the codebook, and if they are close enough then we calculate their average with respect to codebook vector's weight and store it instead of the original vector in the codebook. If we do not find codebook vector close enough to the new vector and the codebook is not full, we add it to the codebook. If the codebook is full, we remove LRU (Last Re- cently Used) vector. This algorithm is intended to replace LBG algorithm to improve compression performance.

\section{3D Vector Quantization for Teleconferencing Video / Confocal Microscopic Images}

The model introduced in this study is based on specific properties such as:

1. History: In teleconferencing and other medical image sequences typical motions of recent history are likely to repeatedly appear in the next frames. History parameter $K_{P}$ relates to the typical length of cache movement. Since $K_{P}$ directly relates to the acceptable delay of the teleconferencing system, it should be determined according to the particular purpose of communication.

2. Localization: The movements are primarily likely to reappear in the same area of the image where they have previously appeared [14].

3. Transformation: If the movements are not similarly repeated (up to the allowed distortion of usually $25-40 \mathrm{db}$ ), details can be encoded as transformed versions of previous details [15].

4. Hierarchy: If a specific motion cannot be matched accurately according to coarse partitioning of the frame, a more refined description should be used $[16,17]$. The hierarchical approach introduces additional parameters, mainly the factor by which the size of $A_{L}$ is changed between adjacent levels of the pyramidal representation. A factor of 2 is used in the present study.

We use 3D VQ to exploit the 3D correlations between voxels from intra-bands. It is different from the method using zero tree structure that exploits the correlations between coefficients from inter bands at different scales.

The choice of intra-band coding rather than inter-band coding is based on the observation that the intra-band models capture most of the dependencies between the sub-bands coefficients. Hence exploiting intra-band redundancies offers a better coding gain than exploiting interband redundancies $[18,19]$. The shape of a vector is specified by its width, height and depth. 


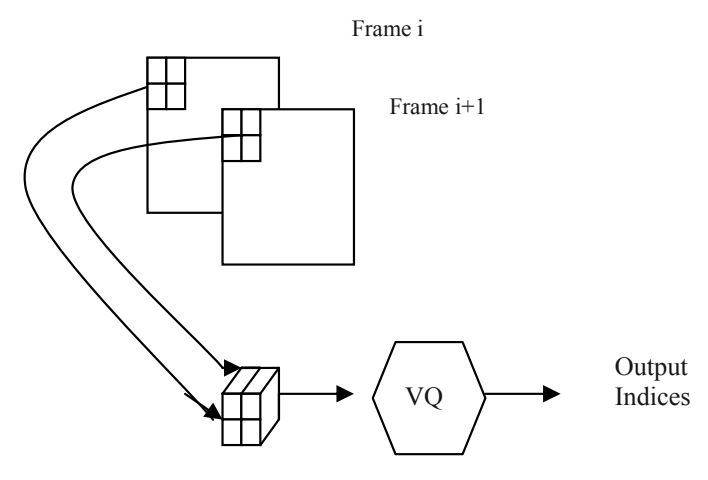

3D Vector

Figure 2. An example of forming an inter-band vector from two successive frames.

For instance, in Figure 2 we construct and eightdimensional vector $2 \times 2 \times 2(w \times h \times d)$ by sampling four neighboring pixels from frame $i$ and four neighboring pixels at the same position from its next frame $i+1$. This choice of vector shape is programmable $[8]$.

\section{Video Compression System and the Encoding Process}

We achieve a very low bit-rate by applying the Vector Quantization (VQ) approach using Adaptive sparse codebooks. Figure 3 shows the block diagram of the system. Frames are divided into frame portions called "super blocks" $(z, y, t)$, each comprising $3 \mathrm{D}$ vectors.

In the above system the super block is $32 \times 32 \times 4$ and the vector shapes are $8 \times 8 \times 4,4 \times 4 \times 4$ arid $2 \times 2 \times 4$. Based on the recent history of the transmitted sequence, a localized codebook that best matches the data is created for each super block shown in Figure 4. Since the recent history of the transmitted sequence is used for training the codebooks, the same codebooks can be created at the remote receiver, hence only an index representing each codebook is transmitted via the transmission medium. A hierarchical

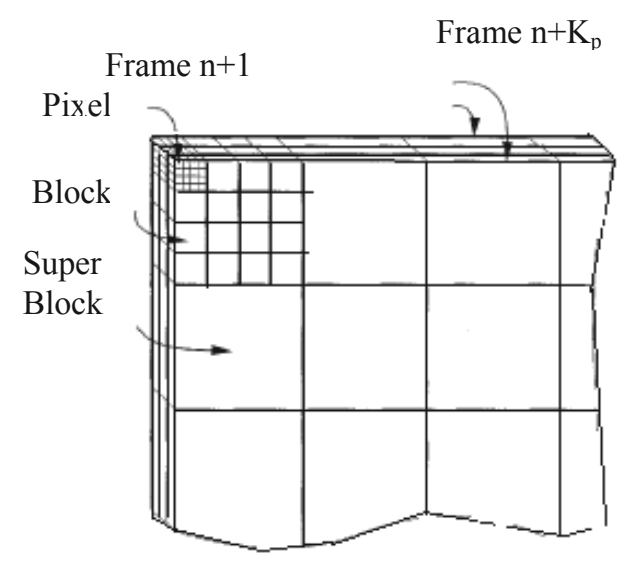

Figure 4. Blocks and Super blocks — each block and each super block contain 4 consecutive frames.

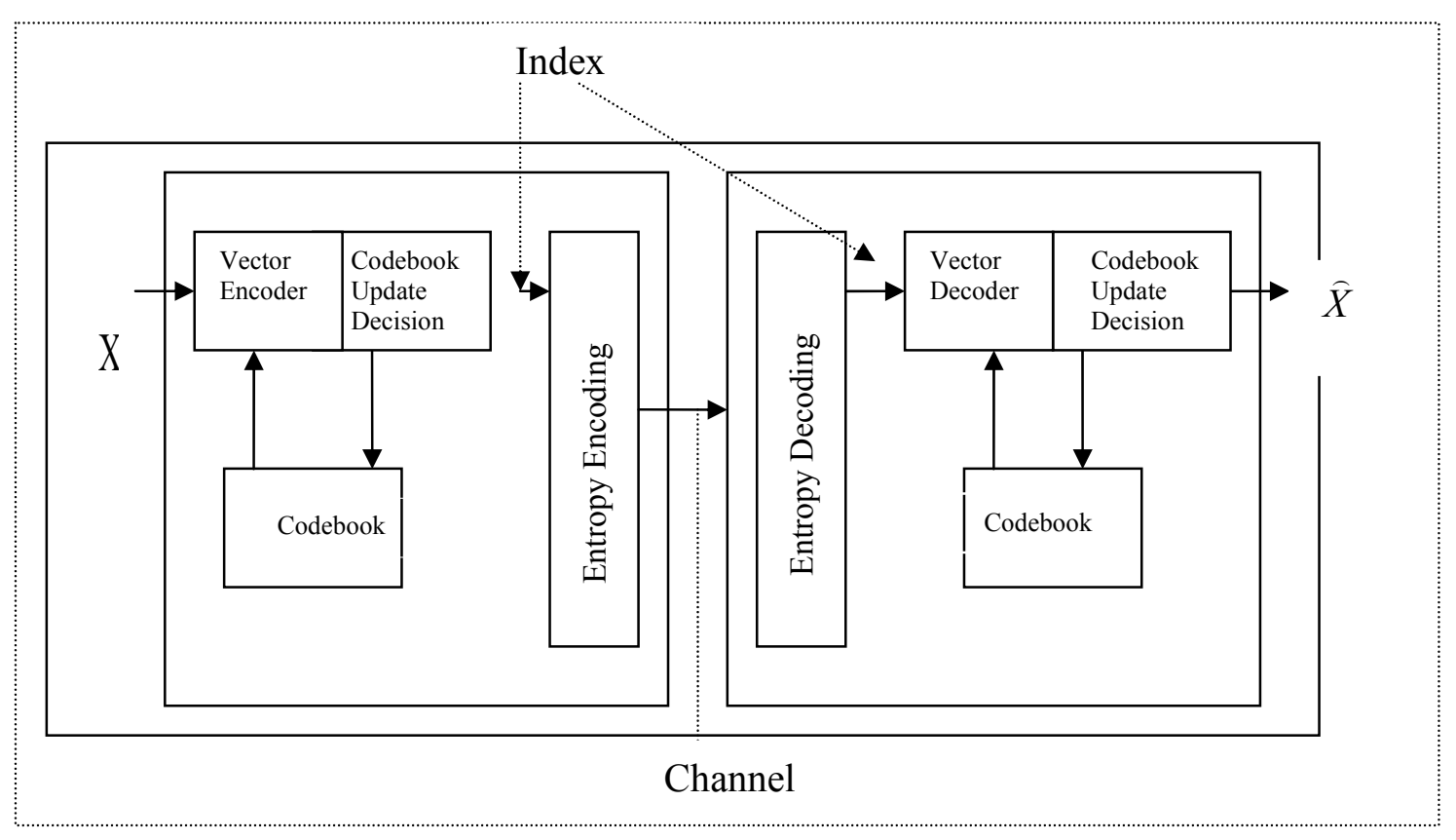

Figure 3. Block diagram of compression system. 


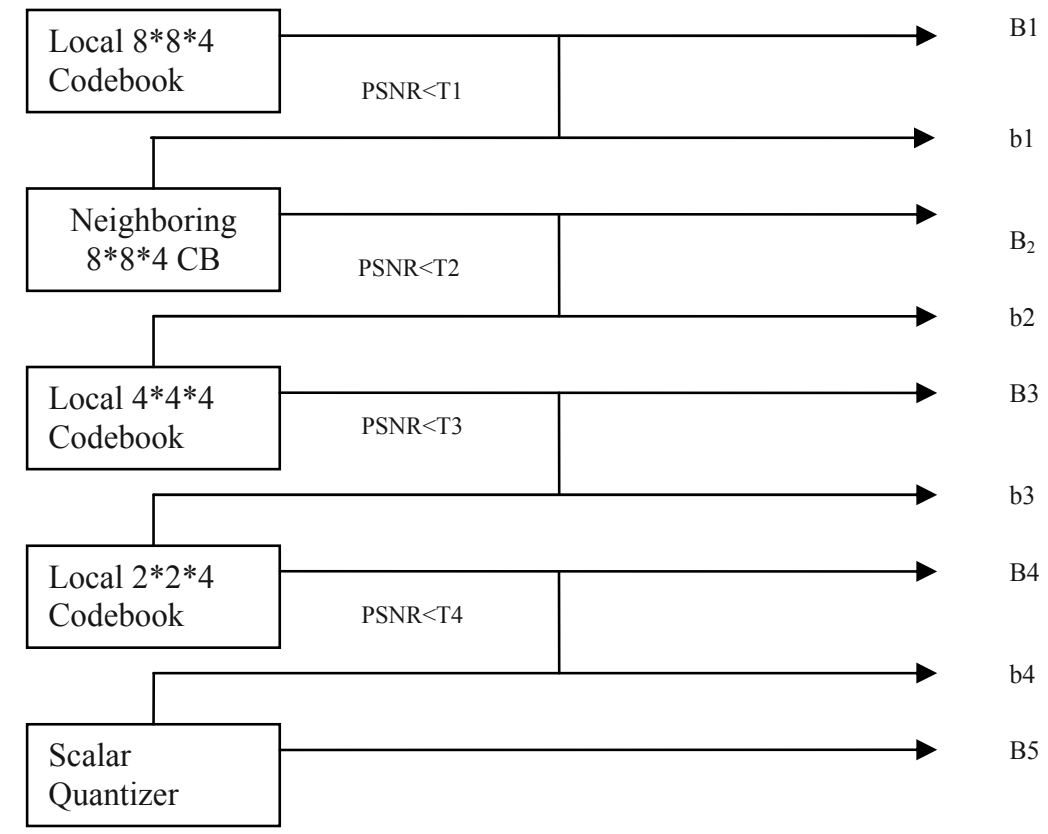

Figure 5. The hierarchical encoding process.

encoding process is performed in the hierarchical encoder. An illustrative encoding process is shown in Figure 5.

The main steps in an encoding process are summarized next.

1. The dedicated localized codebook having the base vector $8 \times 8 \times 4$ from the base history-based VQ is searched. The block having a $P S N R$ greater than threshold $T 1$ is given as output.

2. For the vector with a $P S N R$ less than $T 1$, the search expands to include the codebook of adjacent super block. From this search, vectors having a $P S N R$ greater than $T 2$ are given as output.

3. The vectors unsatisfactorily encoded in 1 and 2 are further coded using more refined codebook of $4 \times 4 \times 4$. Afterwards, a search is made for vectors having a $P S N R$ greater than $T 3$.

4. Remaining blocks are encoded using $2 \times 2 \times 4$ codebook and are output if the encoded vector have a PSNR greater than $T 4$.

5. The vectors inadequately coded using codebook in the above steps are coded by scalar quantization.

Our decoder uses three-dimensional vector-quantization $(x, y, t)$. We encoded the present frames based on the recent localized history of the sequence. In Figure 5, $B 1$ to $B 5$ represent the encoded codes output to the receiver at the respective stage and $b 1$ to $b 4$ represent the control bits for signifying whether the $B 1$ to $B 5$ codes of the next stage are to be used.

Thus the encoder encodes the input image data into various sized codebooks based on the PSNR value of the difference frames. Taking the difference frames for the encoding process instead of direct vectors improves the performance of our system. It enhances the performance and makes the system robust to illumination changes.

\section{An Efficient Codebook Post Processing Technique and a Window Based Fast Search Algorithm for Vector Quantization}

Post processing is done to reorder the codebook according to the codeword potentials. We want the vector quantization to generate more dependent indices without sacrificing the performance. The inter index correlation results from two properties of a vector quantizer:

1) correlation preservation property

2) correlation generation property. 
In other words, the inter index correlation is: a) inherited from the inter vector correlation in the spatial domain and b) generated by the vector quantizer. Our objective is to design a vector quantizer of a high correlation preservation capability. A natural way to achieve this objective is to order the code words in such a manner that 'similar' code words have close indices. In addition, the more similar the code words, the more closely the indices should be. Moreover, an effective measurement of the similarity should be utilized to order the code words. We adopt the potential of a vector as a measure of similarity. The potential of a vector is define as:

$$
P(x)=\sum_{i=1}^{K} W_{i}^{2}
$$

where $W_{i}$ are the values (for pixels) stored in the vector.

The potential here is identical to the energy of the vector. From the definition, we can easily obtain the following equality:

$$
|P(x)-P(\widehat{x})| \leq P(x-\widehat{x}) .
$$

We used the Window-based Fast Search (WBFS) algorithm, which first searches the window built around the predicated index to accelerate the codebook searching procedure.

In Figure 6, vectors $A, B$ and $C$ denote the left, left-above neighboring vectors of current vector $X$, respectively. The horizontal potential gradient $\left(G_{H}\right)$ and vertical potential gradient $\left(G_{v}\right)$ are defined as follows:

$$
G_{v} \equiv\left|P_{A}-P_{B}\right|
$$

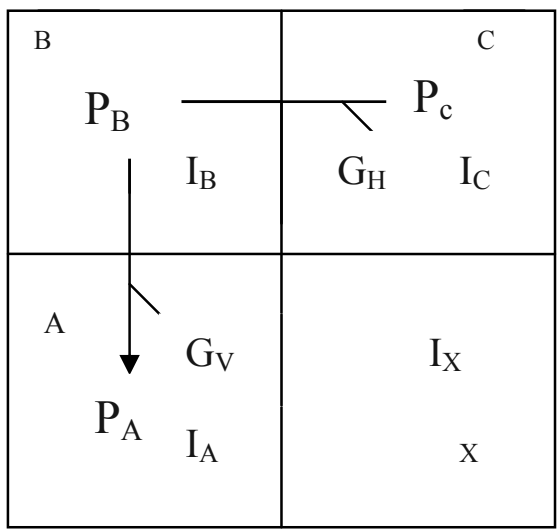

Figure 6. Neighborhood of an input vector $X$ in a frame.

$$
G_{H} \equiv\left|P_{c}-P_{B}\right|
$$

where $P_{A}, P_{B}$ and $P_{C}$ are potentials of vector $A, B$, and $C$ respectively. Then the prediction is made according to the relation:

$$
\begin{aligned}
& \text { if } \quad G_{H} \leq G_{V} \rightarrow \widehat{I}_{X}=I_{A}, \\
& \text { if } \quad G_{H} \geq G_{V} \rightarrow \widehat{I}_{X}=I_{C} .
\end{aligned}
$$

The reordering is done once for all and is applicable to all the existing codebook design methods. The post-processing codebook possesses a special structure which in turn can lead to decreased first-order differential entropy, i.e. to increased correlation between successive indices. This is beneficial to subsequent entropy coding and helps to improve the performance of the entire coding system.

\section{Experimental Results}

The proposed video-compression system was developed and tested using "Salesman" and "Miss America" image sequences. These image sequences contain a moving head of a person in a stable background. Moreover, the performance of this system over microscopic images is tested by using the image sequence "Distribution of GFP in Arabidopsis cells" [19].

In addition to visual inspection, the performance of the proposed compression system was evaluated and compared to that of the existing approaches, using a popular quantitative measure of the image quality known as the peak signalto-noise ratio (PSNR), which is defined as:

$$
\begin{aligned}
& P S N R= \\
& 10 \times \log _{10}\left[\frac{255^{2}}{\frac{1}{N_{1} N_{2}} \sum_{y=1}^{N_{2}} \sum_{x=1}^{N_{1}}(f(x, y)-g(x, y))^{2}}\right]
\end{aligned}
$$

where

-255 is Peak gray level of the frame; 
- $f(x, y) \& g(x, y)$ are the gray levels of pixels from the original and reconstructed frames, respectively.

$-N_{1} \times N_{2}$ is the total number of pixels in the frame.

The degree of compression is measured by the compression ratio defined as:

$$
\text { CompressionRatio }=\frac{\text { OriginalFrameSize }(\text { bytes })}{\text { EncodedFrameSize }(\text { bytes })}
$$

The performance of the proposed video-compression system was compared with that of the ITUT H.261 video-compression standard developed to facilitate video-conferencing and video-phone services over the integrated services digital network (ISDN) [20]. The H.261 standard operates at $1-30 \mathrm{kbits} / \mathrm{s}$.

This coding scheme is a hybrid of inter-frame prediction, transform coding, and motion compensation. Inter-frame prediction removes temporal redundancy while transform coding removes spatial redundancy.

Figure 7 shows $P S N R$ value decreases consistently as the compression ratio increases indicating that the proposed system produces significantly higher compression ratio values than the H.261 standard when the "Miss America" image sequence is encoded at PSNR below 35. The acronym HVQ means Hierarchial Vector
Quantization technique, AC refers to Adaptive codebook and EPPT refers to our Windowsbased Efficient Post-processing Technique. The proposed "post-processing-based video codec" produced significantly higher compression ratio than the H.261 standard on "Miss America" image sequence for any PSNR value.

Figure 8 shows input and output of a frame from 26 to 28 of the "Miss America" video sequence. From the output frames at PSNR 33, it can be seen that the image quality is not very good and frames of this quality cannot be used for medical images. However for teleconferencing video these frames are good because they provide a very high compression ratio. These outputs are obtained by applying VQ+AC+EPP. The visual quality of the image sequences reconstructed by the H.261 standard degraded sharply for high compression ratios, where the proposed system outperforms the H.261 standard.

Figure 9 shows the average compression ratio values computed on the Confocal Microscopic image sequence after its encoding by the proposed system and the LBG-based method at different $P S N R$ values. It is evident that pyramidal codebook-based model with potential-based post-processing on adaptive codebook produces significantly higher compression ratio values than the $3 \mathrm{D}$ vector quantization with $\mathrm{LBG}$ codebook for confocal images.

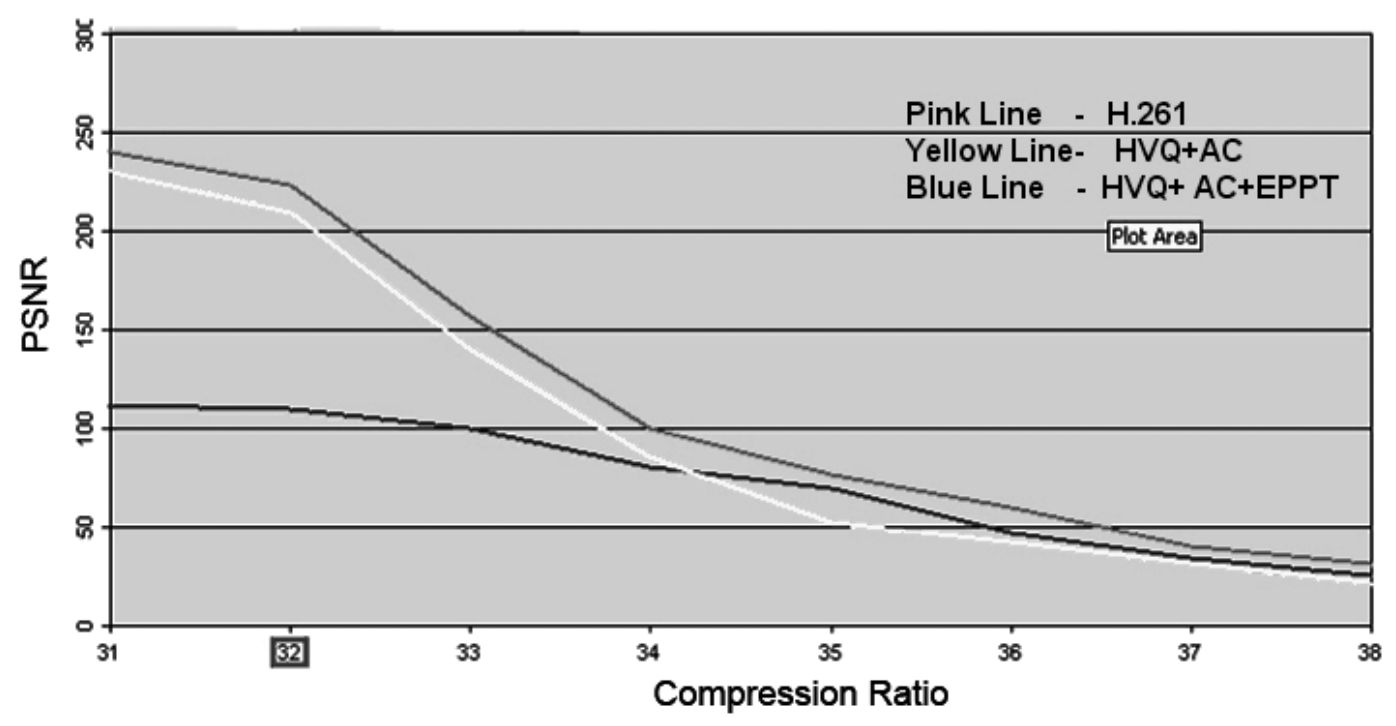

Figure 7. Comparison of the proposed systems with the H.261 video-compression standard on the "Miss America" image sequences. 


\section{Input Frame}
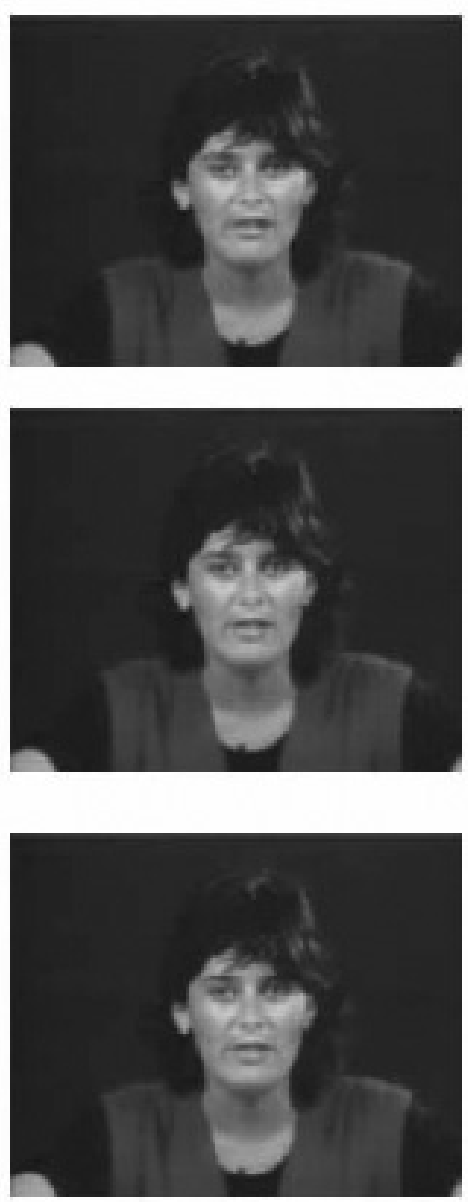

Output Frame
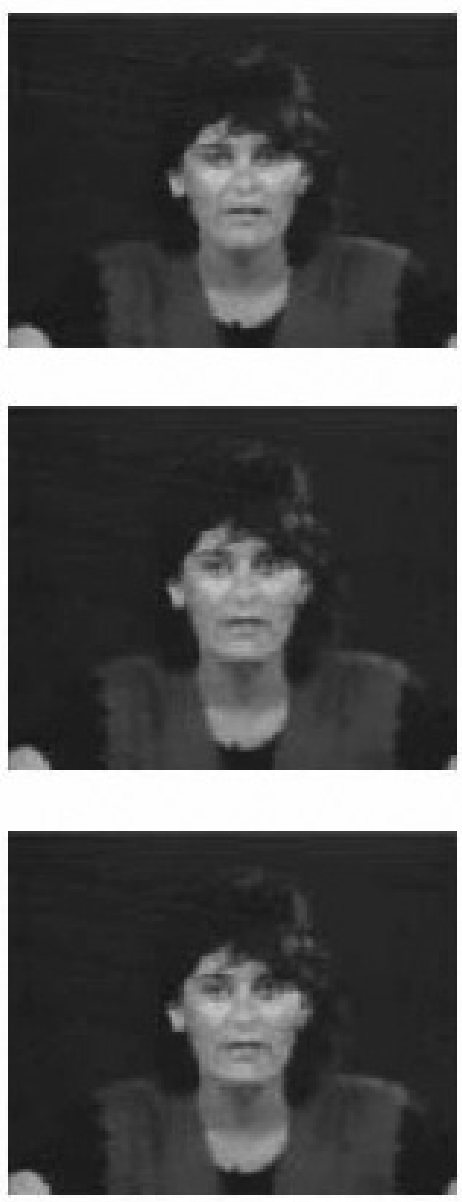

Figure 8. The "Miss America" video sequence at PSNR value 33 and compression ratio 220.

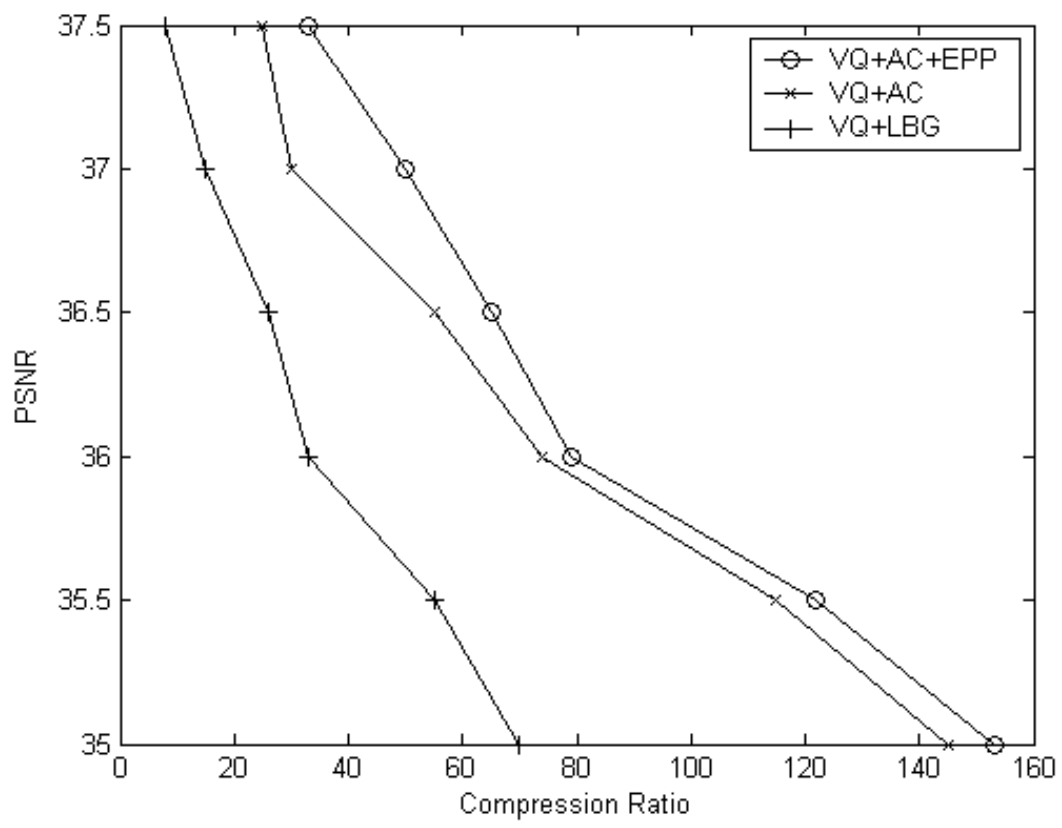

Figure 9. Comparison of the proposed system with LBG-based VQ. 


\begin{tabular}{|l|c|c|c|}
\hline & $\begin{array}{c}\text { Adaptive } \\
\text { CB-based } \\
\text { Hierarchical VQ }\end{array}$ & $\begin{array}{c}\text { Hierarchical + } \\
\text { Adaptive CB } \\
\text { + CB PP }\end{array}$ & H.261 \\
\hline \hline $\begin{array}{l}\text { "Miss } \\
\text { America" }\end{array}$ & 204 & 220 & 110 \\
\hline $\begin{array}{l}\text { Lab } \\
\text { Sequence }\end{array}$ & 160 & 172 & 80 \\
\hline
\end{tabular}

Table 1. Compression ratios at $P S N R=32$.

Input Frame

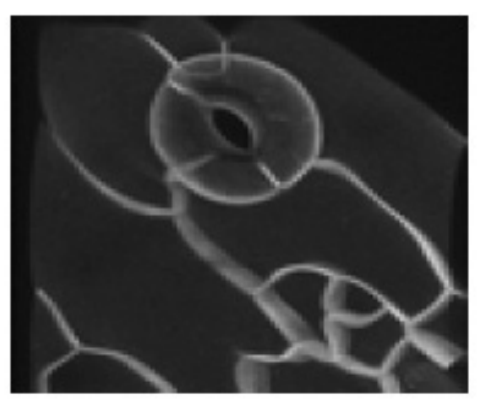

\begin{tabular}{|l|c|c|c|}
\hline & $\begin{array}{c}\text { Adaptive } \\
\text { CB-based } \\
\text { Hierarchical VQ }\end{array}$ & $\begin{array}{c}\text { Hierarchical + } \\
\text { Adaptive CB } \\
\text { + CB PP }\end{array}$ & H.261 \\
\hline \hline $\begin{array}{l}\text { "Miss } \\
\text { America" }\end{array}$ & 21.5 & 31 & 25 \\
\hline $\begin{array}{l}\text { Lab } \\
\text { Sequence }\end{array}$ & 13 & 19 & 16 \\
\hline
\end{tabular}

Table 2. Compression ratios at $P S N R=38$.

\section{Output Frame}

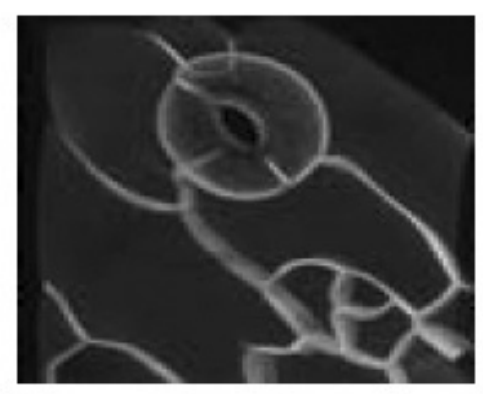

Figure 10. Output for set 1 at compression ratio of 42 and PSNR value of 38.
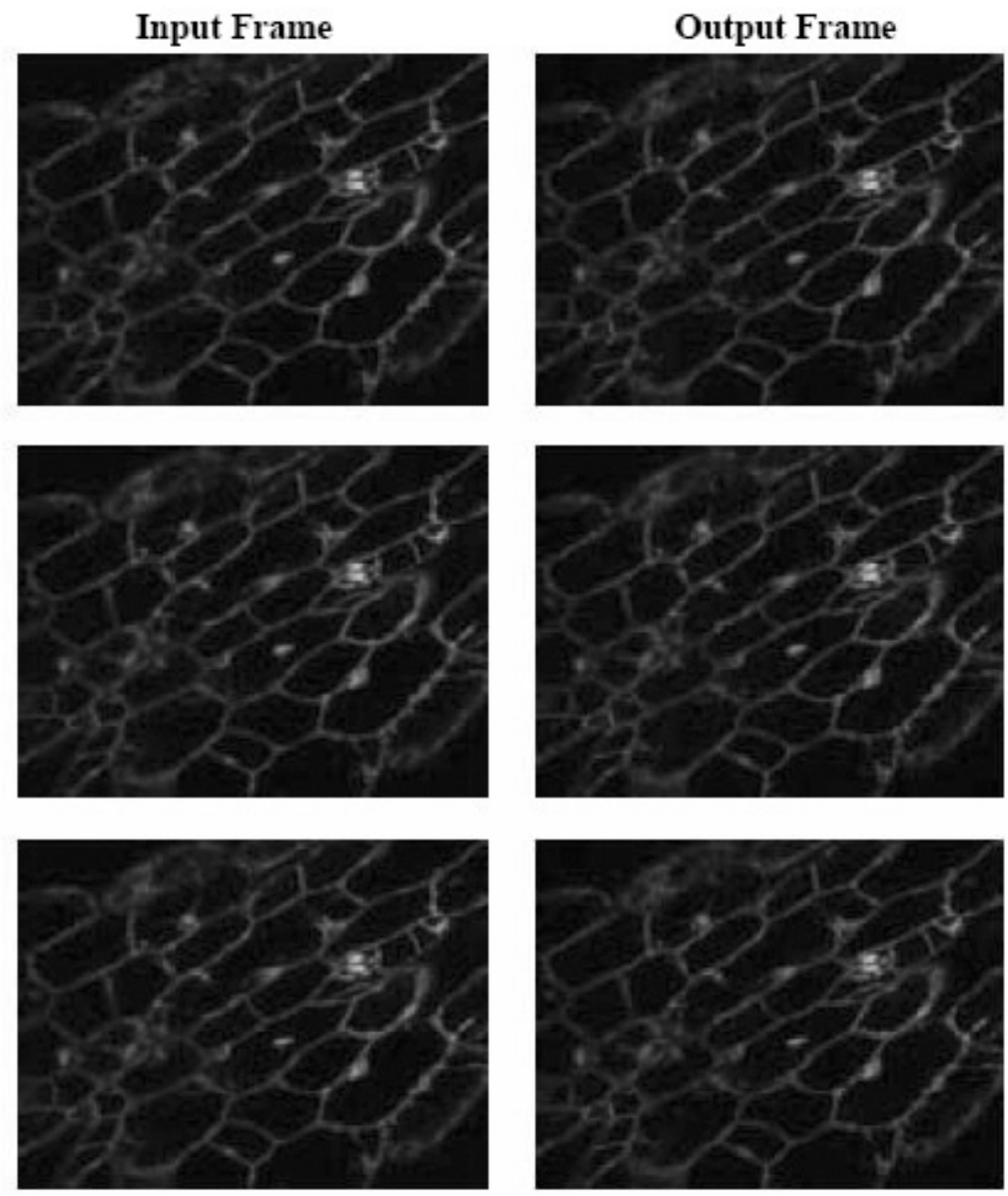

Figure 11. Output at compression ratio of 42 and PSNR value of 38 for set 2. 
Input Frame

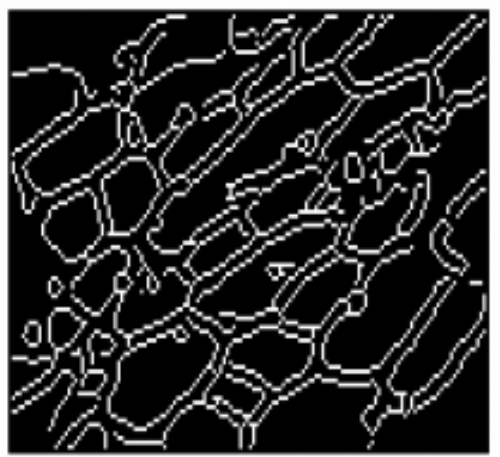

Output Frame

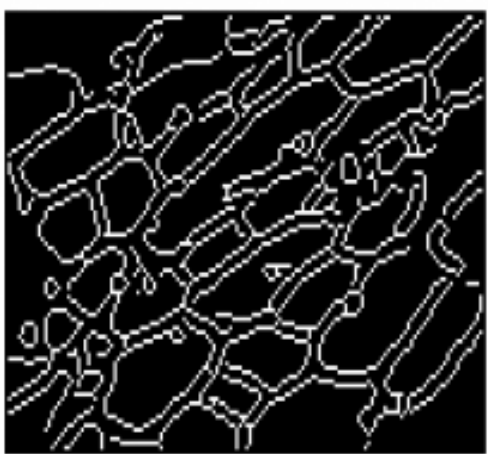

Figure 12. Output at compression ratio of 42 and PSNR value of 38 for edges of set 2 obtained using Canny edge detector.

The tables show the performance comparison of proposed method with H.261. The performance of the proposed video-compression system is compared with that of the LBG codebook-based video compression This coding scheme is vector quantization-based with localized codebook.

Figures 10, 11, and 12 show the input and output frames for confocal microscopic images. The output frames are at PSNR 38. The image quality is very good and there is no perceptual visual difference between these inputs and output frames. These outputs are obtain by applying $\mathrm{VQ}+\mathrm{AC}+\mathrm{EPP}$.

Figure 12 shows that no loss of edges takes place when proposed system is applied on confocal medical images at high PSNR values. Edge preservation is important for medical images. We have used Canny edge detector for detecting edges.

\section{Conclusion and Future Work}

In this paper, a video compression scheme using $3 \mathrm{D}$ vector quantization pyramidal codebook with adaptive codebook is discussed. The approach exploits the fact that microscopy data has limited variations. The performance comparison with coders like H.261 explains the effectiveness of our approach. Unlike other lossy approaches like MPEG-4 or H.263, our approach preserves most of the image data in microscopy images. We also introduced a 3D vector quantization pyramidal codebook-based model with potential-based post-processing on adaptive codebook. The proposed approach outperforms the H.261 teleconferencing video compression standard at high compression ratios. With potential-based post-processing it outperforms H.261 even for low compression ratios. It gave good results for confocal microscopic images and outperformed the LBGbased VQ methods. Moreover, it preserved the edges in confocal images.

Recent works help in achieving both on-the-fly compression and high compression efficiency, which reduces the power dissipation of the device. Such techniques embedded with our adaptive pyramidal codebook approach can be fused together to implement real time cheap telemedicine systems. In addition, faster algorithms for search can be used to make the application more robust.

\section{References}

[1] J. Chou, M. Crouse, K. R., A simple algorithm for removing blocking artifacts in block-transform coded images. IEEE Signal Processing Letters, 5(2) (1998), pp. 33-35.

[2] M. PORAT, Localized Model-based Approach to Teleconferencing using Three-dimensional Vector Quantization. International Conference on Digital Signal Processing, (1997) Greece, pp. 841-844.

[3] A. GERSHO AND R. M. GRAY, Vector Quantization and Signal Compression. Norwell, MA: Kluwer, 1992.

[4] N. M. Nasrabadi, C. Y. ChoO, AND Y. Feng, Dynamic finite-state vector quantization of digital images. IEEE Trans. Commun., 42 (May 1994), pp. 2145-2154. 
[5] C.-H. HsiEH AND J. C. TSAI, Lossless compression of VQ index with search-order coding. IEEE Trans. Image Processing, 5 (Nov. 1996), pp. 1579-1582.

[6] E. H. Adelson, C. H. Anderson, J. R. Bergen, P. J. BURT AND J. M. OGDEN, Pyramid Methods in Image Processing. RCA Engineer, 29 (Nov/Dec 1984), pp. 33-41.

[7] Peter J. Burt and E. H. Adelson, The Laplacian Pyramid as a Compact Image Code. IEEE Trans. Comm., 31 (Apr. 1983), pp. 532-540.

[8] W. P. Cockshott, Y. TaO, G. GaO, P. Balch, A. M. BRIONES, C. DALY, Confocal Microscopic Image Sequence Compression using Vector Quantization and 3D Pyramid. PACS classification number: 07.05.pj, July 15th, 2003.

[9] J. B. PAWLEY, Handbook of Biological Confocal Microscopy, 2nd Edition. Plenum Press, New York, 1995.

[10] C. Daly, A. McGee, E. Vila, A. Briones, J. Giraldo, S. ARribas, C. GonZÁlez, J. M. GonzÁlez, E. SOMOZA, S. N. PAGAKIS, J. ADLER, J. C. Provost, A. Merle, J. Maddison, J. PederSON, AND J. C. MCGRATH, Analysing the 3D Structure of Blood Vessels Using Confocal Microscopy. Microscopy and Analysis (UK), 92 (2002), pp. 5-8.

[11] Y. TAO, W. P. CockshotT, A Hybrid Vector Quantizer for Laplacian Pyramid Coding with Application to Volumetric Image Compression in Confocal Microscopy. SPIE on Photonics Asia 2004, (Nov. 2004) Beijing, China.

[12] P. H. Westernik, D. E. Boekee, J. Biemond AND J. W. Woods, Subband Coding using Vector Quantization. IEEE Trans. Commun., COM-36 (1988), pp. 713-719.

[13] Y. Linde, A. BuZO, R. M. GRAY, An Algorithm for Vector Quantizer Design. IEEE Trans. Commun., 28 (Jan. 1980), pp. 84-95.

[14] M. Porat AND Y. Y. ZEEVI, The Generalized Gabor Scheme in Biological and Machine Vision. IEEE Trans. on Patt. Anal. and Machine Intell., PAMI-10(4) (1988), pp. 452-468.

[15] N. Katzir, M. Lindenbaum And M. Porat, Curze Segmentation under Partial Occlusion. IEEE Trans. on Patt. Anal. and Machine Intell., PAMI-16(5) (1994), pp. 523-519.

[16] M. PORAT AND Y. Y. ZEEVI, Localized Texture Processing in Vision: Analysis and Synthesis in the Gaborian Space. IEEE Trans. on Biomedical Eng., BME-36(1) (1989), pp. 115-129.

[17] T. EBRAhimi, T. R. REED, AND M. KunT, Low Bit Rate Coding of Image Sequences Using a Pyramidal Gabor Expansion. In Proceedings of the 1991 Picture Coding Symposium, (1991) Tokyo, Japan, pp. 205-206.

[18] P. Cosman, R. Gray, And M. VetTerli, Vector Quantization of Image Subbands: A Survey. IEEE Trans. Image Processing, 5 (Feb. 1996), pp. 202225.
[19] P. Schelkens, A. Munteanu, J. Barbarien, M. GalcA, X. G. NiETO, AND J. CORNELIS, Wavelet Coding of Volumetric Medical Datasets. Submitted to IEEE Trans. Medical Imaging, September 28, (2001).

[20] ITU Recommendation H.261: Video codec for audio visual services at $\mathrm{p}^{*} 64 \mathrm{kbits} / \mathrm{second}$, COM XV-R 37-E, 1990.

Received: June, 2006 Revised: January, April, December, 2007 Accepted: August, 2008

Contact address:

Vinay Arya, Ankush Mittal, R. C. Joshi Dept. of Electronics and Computer Engineering IIT Roorkee Uttaranchal, India 247667 e-mail: vin_gs@rediffmail.com ankumfec@iitr.ernet.in joshifec@itr.ernet.in Amit Pande Dept. of Electrical and Computer Engineering Iowa State University Ames, IA-50011, USA e-mail: amit@iastate.edu

VINAY ARYA completed his Masters (M. Tech.) at IIT Roorkee in 2005. His research interests include medical image processing and video coding.

ANKUSH MitTAL received the B. Tech. (Computer Science and Engg.) and M.S. by Research (Computer Science and Engg.) degrees from the Indian Institute of Technology, Delhi in 1996 and 1998 respectively. He completed his PhD degree at Electrical and Computer Engg. Dept, The National University of Singapore. For two years dr. Mittal was a faculty member at the Department of Computer Science, National University of Singapore. He is presently an associate professor at Electronics and Computer Engineering Department at IIT Roorkee. He is a recipient of Young Scientist Award from the National Academy of Sciences, India and from Indian Science Congress Association. He has contributed more than 150 research papers in journals and conferences of high repute. His research interests are in image processing, bioinformatics, Bayesian networks, and applications in e-learning.

AMIT PANDE is currently a graduate student at Iowa State University, USA pursuing his research in developing efficient multimedia processing reconfigurable hardware architectures. He completed his undergraduation at IIT Roorkee where their major project was awarded with Institute Silver Medal, honored as third best major project in agilent engineering and with Technology Awards (India) in 2007. His research interests are in image processing, e-learning and applications in computer architecture.

R. C. JosHI received his B.E. degree in electrical engineering from Allahabad University in 1967. He received his M.E. and Ph.D. degrees in electronics and computer engineering from the University of Roorkee in 1970 and 1980, respectively. He has published about 50 research papers in national/international journal/conferences. He has also received a Gold Medal from the Institute of Engineers in 1978 for the best research paper. He was also a member of National Industrial Research and Development Award Committee. He has delivered about 20 special lectures at various US and Indian universities and organizations (University of Minnesota, Ohio University, Wayne State University, North Carolina University, Florida Atlantic University, etc.). 